\title{
Professor Dr. med. Heinrich Matthys zum 75. Geburtstag
}

\author{
Professor Dr. med. Heinrich Matthys on the Occasion of his $75^{\text {th }}$ Birthday
}

Autoren

Institute
J. Müller-Quernheim, S. Sorichter

Abteilung für Pneumologie, Medizinische Klinik, Albert-Ludwigs-Universität Freiburg
Bibliografie

Dol http://dx.doi.org/ $10.1055 / \mathrm{s}-0029-1243987$ Pneumologie 2010; 64: 259-259 @ Georg Thieme Verlag KG Stuttgart · New York ISSN 0934-8387

\section{Korrespondenzadresse}

Prof. Dr. med.

J. Müller-Quernheim

Ärztlicher Direktor

Abteilung Pneumologie

Medizinische Klinik

Universtitätsklinikum Freiburg

Killianstr. 5

79106 Freiburg

joachim.mueller-quernheim@

uniklinik-freiburg.de
Über 25 Jahre - von 1975 bis 2002 - leitete Heinrich Matthys die Abteilung für Pneumologie der Medizinischen Klinik der Albert-Ludwigs-Universität Freiburg i. Br. In dieser Zeit war er einer derjenigen, die in Deutschland das Fach Pneumologie prägten. Daher ist sein Name nach wie vor untrennbar mit dem Fach verbunden.

Er ist einer der Väter der klinischen Atemphysiologie. Seine Arbeiten zu den Normwerten und zur klinischen Interpretation bilden noch heute die Basis der täglichen Befundung und neuer Befundalgorithmen. Die biomedizinischen Fragen, die mit diesen Methoden bearbeitet werden konnten, sind im Wesentlichen noch während der Jahre seiner aktiven Berufstätigkeit bearbeitet worden. Er hatte den Weitblick, die Grundsteine für neue Entwicklungen zu legen, und zum Beispiel die Analyse der Atempumpe findet jetzt in der täglichen Routine zunehmend Bedeutung, was nicht zuletzt auf seine Förderung dieses Themas zurückzuführen ist.

Sein 1982 erstmals erschienenes Lehrbuch „Klinische Pneumologie“ hat sich zu einem deutschsprachigen Standardwerk entwickelt. 2008 ist nun die vierte Auflage erschienen und hier stellen insgesamt 60 Autoren aus dem deutschsprachigen Raum den aktuellen Stand der Pneumologie in seiner gesamten Breite dar. Seine große Erfahrung im Wissenschaftsbetrieb hat er der European Respiratory Society zur Verfügung gestellt, in den letzten Jahren hat er viele Ämter in der Selbstver-

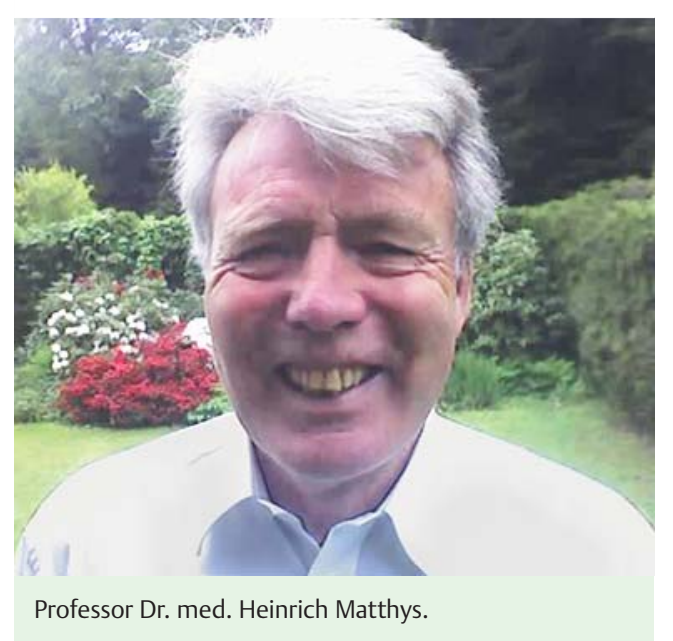

waltung dieser Gesellschaft übernommen und so weiterhin die Entwicklung der Pneumologie in Europa mitgestaltet.

Lieber Heinrich, wir wünschen Dir, auch im Namen Deiner Freunde, Kollegen und Schüler, zum 75. Geburtstag alles Gute und noch viele gesunde Jahre. Wir hoffen, dass der vielbeschäftigte Großvater auch weiterhin Zeit für die Pneumologie finden wird.

J. Müller-Quernheim, S. Sorichter Freiburg 